\title{
Article \\ Infection Prevention Performance among In-Flight Cabin Crew in South Korea
}

\author{
Jaegeum Ryu ${ }^{1,2} \mathbb{D}$, Jungha Kim ${ }^{3, *} \mathbb{D}$ and Smi Choi-Kwon ${ }^{1,2}$ \\ 1 College of Nursing, Seoul National University, Seoul 03080, Korea; ryu301@snu.ac.kr (J.R.); \\ smi@snu.ac.kr (S.C.-K.) \\ 2 The Research Institute of Nursing Science, Seoul National University, Seoul 03080, Korea \\ 3 Aeromedical Center, KoreanAir, Seoul 07505, Korea \\ * Correspondence: dsmgkwjdgk@snu.ac.kr
}

Citation: Ryu, J.; Kim, J.; Choi-Kwon, S. Infection Prevention Performance among In-Flight Cabin Crew in South Korea. Int. J. Environ. Res. Public Health 2021, 18, 6468. https:// doi.org/10.3390/ijerph18126468

Academic Editor: Paul B. Tchounwou

Received: 31 March 2021

Accepted: 12 June 2021

Published: 15 June 2021

Publisher's Note: MDPI stays neutral with regard to jurisdictional claims in published maps and institutional affiliations.

Copyright: (c) 2021 by the authors. Licensee MDPI, Basel, Switzerland. This article is an open access article distributed under the terms and conditions of the Creative Commons Attribution (CC BY) license (https:// creativecommons.org/licenses/by/ $4.0 /)$.

\begin{abstract}
COVID-19 was declared a worldwide pandemic in 2020; thus, preventing in-flight infection transmission is important for stopping global spread via air travel. Infection prevention (IP) performance among aircraft cabin crew is crucial for preventing in-flight transmission. We aimed to identify the level of IP performance and factors affecting IP performance among aircraft cabin crew during the COVID-19 pandemic in South Korea. An online survey was conducted with 177 cabin crew members between August and September 2020. The survey assessed IP performance, and IP awareness, using a five-point Likert scale, and also evaluated simulation-based personal protective equipment (PPE) training experience, and organizational culture. The average IP performance score was $4.56 \pm 0.44$. Although the performance level for mask-wearing was high (4.73 \pm 0.35$)$, hand hygiene $(\mathrm{HH})$ performance $(4.47 \pm 0.56)$ was low. Multivariate analysis showed that IP performance was significantly associated with IP awareness $(p<0.05)$ and simulation-based PPE training experience $(p<0.05)$. Since $\mathrm{HH}$ performance was relatively low, cabin crew and airlines should make efforts to improve HH performance. Furthermore, a high level of IP awareness and PPE training experience can improve IP performance among cabin crew members. Therefore, simulation-based PPE training and strategies to improve IP awareness are essential for preventing in-flight infection transmission.
\end{abstract}

Keywords: COVID-19; pandemic; infection control; inflight transmission; aircraft

\section{Introduction}

Coronavirus disease 2019 (COVID-19) was first identified as pneumonia of unknown cause in China in December 2019 [1], and has since spread rapidly worldwide [2]. South Korea reported its first COVID-19 infection at the beginning of the global spread, due to the country's close geographical proximity to China [3].

COVID-19 was largely spread from China through people traveling via aircraft, leading to community transmission in other countries [4]. Strong measures, such as border closures, air traffic restrictions, and citywide lockdowns have been implemented to stop the spread of COVID-19 within and between countries [5]. As borders closed, it became difficult for citizens in foreign countries to return home, to which some countries responded by operating national chartered flights to bring back their own citizens [6,7].

However, air travel was reported to have accelerated the H1N1 novel influenza pandemic in 2009. Therefore, in-flight infection prevention (IP) was crucial to stop the spread of the COVID-19 pandemic [8]. Effective strategies suggested to prevent infectious disease associated with air travel include entry screening, isolation, quarantine, and personal hygiene [9]. During a flight, viral transmission could occur by inhaling suspended infectious droplets or through direct contact with an unidentified person with COVID-19 who was asymptomatic while onboard the aircraft $[10,11]$. Moreover, people moving through the corridor of a confined cabin space, such as when cabin crew members provide in-flight 
service, could increase the infection risk $[12,13]$. To prevent the in-flight transmission of COVID-19, cabin crew members are advised to perform IP behaviors, such as using hand sanitizer for thorough hand hygiene $(\mathrm{HH})$ and wearing appropriate personal protective equipment (PPE), such as N95 facemasks [14,15]. N95 facemasks have been reported to significantly reduce the risk of droplet transmission among cabin crew members and passengers onboard aircraft [16], and mass COVID-19 events on flights were rapidly reduced when mandatory mask-wearing was implemented on aircraft due to the significant transmission from pre-symptomatic and symptomatic individuals [17].

Previous studies have reported that IP awareness is related to IP performance [18,19]. For example, improved IP awareness has been shown to increase the intention to comply with IP guidelines among healthcare workers (HCWs) [20]. In particular, in 2015, South Korea experienced an outbreak of another type of coronavirus disease, Middle East Respiratory Syndrome (MERS), which necessitated that HCWs increase their IP awareness [21,22]. After the MERS outbreak, people came to recognize the importance of infection control measures such as hand hygiene and mask-wearing to prevent the spread of infectious disease in South Korea [22]. Along with IP awareness, previous research has indicated that simulation-based PPE putting on and removing may be related to improved IP performance [23]. Realistic and actual practice in properly putting on and removing PPE can ensure that HCWs improve their IP awareness in order to prepare for future uncertain situations during a pandemic $[23,24]$.

Another factor reported to influence IP performance is organizational culture [25]. Organizational culture has been found to improve IP performance in ensuring safety against healthcare-associated infections [26]. In South Korea, organizational culture tends to be more authoritative and collectivist than in Western cultures [27,28]. Additionally, among cabin crew, a collectivist culture has been reported to be fostered due to job characteristics related to ensuring passengers' in-flight service satisfaction and safety in a limited cabin space [29].

Thus, it is essential for cabin crew to engage in IP performance to prevent global transmission of infectious diseases via air travel. Further, it is necessary for cabin crew to strictly adhere to IP guidelines, due to possible exposure to various infectious agents during a flight. However, to the best of our knowledge, IP performance among cabin crew has not been investigated. Moreover, the factors that affect IP performance among cabin crew have not yet been clarified. Therefore, we aimed to increase understanding of the status of IP performance by aircraft cabin crew during the COVID-19 pandemic to identify the factors that affect IP performance.

\section{Materials and Methods}

\subsection{Study Design}

This was a cross-sectional, descriptive, investigative study conducted to better understand the level and related factors of IP performance among cabin crew during the COVID-19 pandemic. This study was conducted after receiving approval from the Institutional Review Board of Seoul National University (SNU 20-07-039).

\subsection{Participants and Data Collection}

This study was conducted with 184 cabin crew members of a major South Korean airline. Participants without in-flight service experience during the COVID-19 pandemic were excluded. Data were collected between August and September 2020, using an online survey (http:/ / en.surveymonkey.com accessed on 29 August 2020). All participants were informed of the study's purpose, content, and data collection procedures, and assured that their information would remain confidential and the collected data would not be used for purposes other than the study. Participants could only complete the online survey after providing their consent to participate in the study. Although the possibility of infection may differ depending on factors such as the type of aircraft, number of passengers, and flight time, the airline included in this study was operating the standard IP procedures at 
the time of data collection. The air inside each aircraft of this airline is circulated every 2-3 min through HEPA filters, and the cabin crew members are asked to wear masks, goggles, gloves, and gowns and to apply enhanced IP procedures during each flight. [30].

\subsection{Measurement Variables}

\subsubsection{General Participant Characteristics}

Data were collected on the participants' general characteristics, including gender, age, marital or partnership status, educational background, job position, and employment duration. We investigated whether the participants had previous experience handling passengers with confirmed or suspected cases of MERS or COVID-19 and quarantining after exposure to these diseases. Regarding COVID-19, according to Korean CDC guidelines, having quarantine experience also meant taking a test for COVID-19 if the participants had come in contact with infected persons or showed symptoms characteristic of COVID-19.

\subsubsection{IP Performance}

To measure IP performance among cabin crew, we modified a tool developed by Hong et al. [31], which was originally developed to measure performance of standard precautions in nursing college students. The questionnaire comprised three areas with a total of 22 questions: $\mathrm{HH}$ (10 questions), mask-wearing (6 questions), and management of passengers suspected of having COVID-19 (6 questions). Participants responded using a five-point Likert scale (never performed $=1$, rarely performed $=2$, sometimes performed $=3$, often performed $=4$, always performed $=5$ ). A higher value indicates higher performance. If participants had no experience with a particular item, they were asked to respond with "never experienced."

We measured the tool's content validity index (CVI) based on three infection control nurses, two airline nurses, and one cabin crew member, all of whom recorded an item-level CVI (I-CVI) of 0.78 or higher and a scale-level CVI/average (S-CVI/Ave) of 0.92, which are considered suitable [32]. The original tool [31] had a Cronbach's $\alpha$ value of 0.95 , whereas the tool used in our study had a value of 0.89 .

\subsubsection{IP Awareness}

To measure IP awareness among cabin crew, we modified a measurement tool developed by Hong et al. [31] comprising 22 questions covering the same three areas as the IP performance measurement tool, with items distributed in the same manner. Participants responded using a five-point Likert scale (not at all important $=1$, not important $=2$, neutral $=3$, important $=4$, and very important $=5$ ); the higher the score, the higher is the awareness.

As with the IP performance measurement, the CVI of this questionnaire was assessed by three infection control nurses, two airline nurses, and one cabin crew, all of whom recorded an I-CVI of 0.78 or higher and an S-CVI/Ave of 0.97, which are considered suitable [32]. The original tool [31] had a Cronbach's $\alpha$ value of 0.97 , whereas the tool used in our study had a value of 0.82 .

\subsubsection{Organizational Culture}

To measure organizational culture, we modified a tool originally developed by Kim [33] to measure organizational culture in hospitals. It consists of seven questions, to which the participants respond using a seven-point Likert scale (strongly disagree $=1$, often disagree $=2$, slightly disagree $=3$, neutral $=4$, slightly agree $=5$, mostly agree $=6$, strongly agree $=7$ ); the higher the value, the better is the organizational culture.

The CVI was measured based on three infection control nurses, two airline nurses, and one cabin crew member, all of whom recorded an I-CVI of 0.78 or higher and an S-CVI/Ave of 0.91 , which are considered suitable [32]. The original tool [33] had a Cronbach's $\alpha$ value of 0.85 , whereas the tool used in our study had a value of 0.80 . 


\subsubsection{Simulation-Based PPE Training}

Since PPE training experience has been shown to be related to improved IP performance, we also evaluated whether participants had previously received simulation-based PPE training. The airline provided guidelines for putting on and taking off PPE to the cabin crew online; however, to ensure IP performance, cabin crew members received simulation-based PPE training by an epidemiological intelligence service officer before boarding COVID-19-related chartered flights to Wuhan, China, and Milan, Italy in January and March 2020, respectively.

\subsection{Statistical Analysis}

Data were analyzed using SPSS (version 25.0; IBM Corp.). Participants' general characteristics were analyzed using descriptive statistics (frequency, percentage, mean, and standard deviation), whereas their IP awareness and performance were analyzed using means and standard deviations. The difference between IP awareness and performance was analyzed using a paired $t$-test. IP performance and general characteristics were analyzed using independent $t$-tests or analysis of variance (ANOVA). The relationships between simulation-based PPE training experience and actual experience handling passengers with, or quarantining due to exposure to, a suspected or confirmed case of MERS and/or COVID19 were analyzed using the Mann-Whitney $U$ test $(n<30$ cases). Correlations between IP performance, IP awareness, and organizational culture were analyzed using Pearson's correlation coefficients. The factors affecting IP performance were analyzed using linear regression analysis with the bootstrap method, which is useful for data that do not meet the assumption of error normality [34]. For all measurement tools, reliability was analyzed using Cronbach's $\alpha$. The level of statistical significance was set at $p<0.05$.

\section{Results}

\subsection{Participant Characteristics}

Of the 184 cabin crew members who agreed to participate in the study, 177 (96\%) were included in the analysis. The participants included 133 women $(75.1 \%), 131$ college graduates $(74.0 \%)$, and 105 general flight attendants (59.3\%). Their average age was $37.58 \pm 8.46$ years, the largest proportion comprising people in their $40 \mathrm{~s}(n=69 ; 39.0 \%)$. The average working experience was $6.26 \pm 3.38$ years. There were $25(14.1 \%)$ and 14 participants $(7.9 \%)$ with experience of handling passengers with suspected and confirmed cases of COVID-19 and MERS in 2015, respectively. There were $19(10.7 \%)$ and $3(1.7 \%)$ participants with quarantine experience due to coming in contact with passengers with suspected and confirmed cases of COVID-19 or MERS (in 2015), respectively. There were 23 participants (13.0\%) who had simulation-based PPE training experience (Table 1).

\subsection{IP Performance and Awareness}

The average level of IP performance was $4.56 \pm 0.44$. The subcategory with the highest score was handling passengers with suspected or confirmed cases of COVID-19 (4.90 \pm 0.41$)$, followed by mask-wearing $(4.73 \pm 0.35)$ and $\mathrm{HH}(4.47 \pm 0.56)$. The average level of IP awareness was $4.75 \pm 0.28$. The subcategory with the highest score was handling passengers with suspected or confirmed cases of COVID-19 (4.97 \pm 0.08$)$, followed by mask-wearing $(4.78 \pm 0.35)$ and $\mathrm{HH}(4.61 \pm 0.45)$.

Upon comparison, the level of IP performance $(4.56 \pm 0.44)$ was significantly lower $(p<0.05)$ than that of IP awareness $(4.75 \pm 0.28)$. By subcategory, the level of performance for $\mathrm{HH}(4.47 \pm 0.56)$ was significantly lower than that of awareness $(4.61 \pm 0.45 ; p<0.05)$ (Table 2). 
Table 1. General characteristics of participants.

\begin{tabular}{|c|c|c|}
\hline \multicolumn{3}{|c|}{$(N=177)$} \\
\hline Variables & Categories & $\mathrm{M} \pm \mathrm{SD}$ or $n(\%)$ \\
\hline \multirow[t]{2}{*}{ Gender } & Female & $133(75.1)$ \\
\hline & Male & $44(24.9)$ \\
\hline \multirow[t]{5}{*}{ Age (years) } & & $37.58 \pm 8.46$ \\
\hline & $20 \sim 29$ & $45(25.4)$ \\
\hline & $30 \sim 39$ & $57(32.2)$ \\
\hline & $40 \sim 49$ & $69(39.0)$ \\
\hline & $50 \sim 59$ & $6(3.4)$ \\
\hline Marriage & Married & $67(49.2)$ \\
\hline Living with & Alone & $35(19.8)$ \\
\hline \multirow[t]{3}{*}{ Education } & College & $27(15.3)$ \\
\hline & University & $131(74.0)$ \\
\hline & Graduate or above & $19(10.7)$ \\
\hline \multirow[t]{2}{*}{ Position } & Team leader & $72(50.7)$ \\
\hline & Staff & $105(59.3)$ \\
\hline Working period (years) & & $6.26 \pm 3.38$ \\
\hline \multirow[t]{2}{*}{ Experience handling passengers with } & MERS $^{1}$ & $14(7.9)$ \\
\hline & COVID $-19^{2}$ & $25(14.1)$ \\
\hline \multirow[t]{2}{*}{ Quarantined due to contact with } & MERS $^{1}$ & $3(1.7)$ \\
\hline & COVID $-19^{2}$ & $19(10.7)$ \\
\hline \multicolumn{2}{|c|}{ Simulation-based $\mathrm{PPE}^{3}$ training } & $23(13.0)$ \\
\hline
\end{tabular}

Table 2. Awareness and performance of infection prevention in participants.

\begin{tabular}{|c|c|c|c|c|}
\hline \multicolumn{5}{|c|}{$(N=177)$} \\
\hline \multirow{2}{*}{ Categories } & \multicolumn{2}{|c|}{$\mathbf{M} \pm \mathbf{S D}$} & \multirow{2}{*}{$t$} & \multirow{2}{*}{$p$} \\
\hline & Awareness & Performance & & \\
\hline Hand hygiene & $4.61 \pm 0.45$ & $4.47 \pm 0.56$ & 3.86 & $<0.05$ \\
\hline Wearing a mask & $4.78 \pm 0.35$ & $4.73 \pm 0.35$ & 1.72 & 0.088 \\
\hline Handling passengers with confirmed or suspected COVID-19 1 & $4.97 \pm 0.08$ & $4.90 \pm 0.41$ & 0.96 & 0.347 \\
\hline Average & $4.75 \pm 0.28$ & $4.56 \pm 0.44$ & 6.54 & $<0.05$ \\
\hline
\end{tabular}

${ }^{1}$ COVID-19 = coronavirus disease 2019.

\subsection{Factors Related to IP Performance}

A univariate analysis showed that simulation-based PPE training experience $(p<0.05)$, IP awareness $(p<0.05)$, and organizational culture $(p<0.05)$ affected IP performance. The performance level of cabin crew members with simulation-based PPE training experience ( $n=23$, median $=4.76$, range: $3.33 \sim 5.00)$ was significantly higher than that of cabin crew members without such experience $(n=154$, median $=4.64$, range: $2.79 \sim 5.00)$. The performance of cabin crew members who experienced MERS in $2015(n=14$, median $=4.87$, range: $4.19 \sim 5.00)$ was higher than that of those who had no such experience $(n=163$, median $=4.67$, range: $2.79 \sim 5.00)$; however, the difference was not statistically significant $(p=0.078$; Table 3$)$.

The multivariate analysis showed that IP awareness $(B=0.77, p<0.05)$ and simulationbased PPE training experience $(B=0.25, p<0.05)$ significantly affected cabin crew members' IP performance. The regression model had an explanatory power of $29 \%$, which corresponded to the medium effect size as given by Cohen [35,36], and an appropriate Durbin-Watson d value of 1.97. All variance inflation factors were less than 10, thereby ruling out multi-collinearity [37]. We used the bootstrap method to perform a linear regression analysis, as the assumptions of multivariate normality, linearity, and normally 
distributed errors were not met (Kolmogorov-Smirnov normality test $Z=1.95, p<0.05$; Breusch Pegan test $\chi^{2}=16.80, p<0.05$; Table 4) [34].

Table 3. Univariate analysis of factors affecting infection prevention performance.

\begin{tabular}{|c|c|c|c|c|c|}
\hline \multicolumn{6}{|c|}{$(N=177)$} \\
\hline \multirow{2}{*}{ Variables } & \multirow{2}{*}{\multicolumn{2}{|c|}{ Categories }} & \multicolumn{3}{|c|}{ Performance $^{5}$} \\
\hline & & & $\mathrm{M} \pm \mathrm{SD}$ or Median (Range) & $t, F, Z$, or $\mathbf{r}$ & $p$ \\
\hline \multirow{2}{*}{ Gender } & \multicolumn{2}{|c|}{ Female } & $4.55 \pm 0.47$ & -0.86 & 0.393 \\
\hline & & & $4.61 \pm 0.33$ & & \\
\hline \multirow{4}{*}{ Age (years) } & \multicolumn{2}{|c|}{ 20 29 } & $4.55 \pm 0.44$ & 0.60 & 0.616 \\
\hline & \multicolumn{2}{|c|}{$30 \sim 39$} & $4.52 \pm 0.42$ & & \\
\hline & \multicolumn{2}{|c|}{$40 \sim 49$} & $4.58 \pm 0.45$ & & \\
\hline & \multicolumn{2}{|c|}{$50 \sim 59$} & $4.76 \pm 0.33$ & & \\
\hline \multirow{3}{*}{ Marriage } & \multicolumn{2}{|c|}{ Single } & $4.58 \pm 0.42$ & 0.54 & 0.592 \\
\hline & \multicolumn{2}{|c|}{ Married } & $4.55 \pm 0.45$ & & \\
\hline & \multicolumn{2}{|c|}{ College } & $4.56 \pm 0.49$ & 0.04 & 0.965 \\
\hline \multirow[t]{2}{*}{ Education } & \multicolumn{2}{|c|}{ University } & $4.57 \pm 0.43$ & & \\
\hline & \multicolumn{2}{|c|}{ Graduate or above } & $4.54 \pm 0.38$ & & \\
\hline \multirow{2}{*}{ Position } & \multirow{2}{*}{\multicolumn{2}{|c|}{$\begin{array}{l}\text { Team leader } \\
\text { Staff }\end{array}$}} & $4.57 \pm 0.43$ & -0.09 & 0.928 \\
\hline & & & $4.56 \pm 0.44$ & & \\
\hline \multirow{4}{*}{ Experience handling passengers with } & \multirow{2}{*}{ MERS $^{1}$} & Yes & $4.87(4.19,5.00)$ & -1.76 & 0.078 \\
\hline & & No & $4.67(2.79,5.00)$ & & \\
\hline & \multirow{2}{*}{ COVID-19 2} & Yes & $4.76(3.33,5.00)$ & -1.21 & 0.227 \\
\hline & & No & $4.65(2.79,5.00)$ & & \\
\hline \multirow{4}{*}{ Quarantined due to contact with } & \multirow{2}{*}{ MERS $^{1}$} & Yes & $4.73(3.53,5.00)$ & -0.03 & 0.979 \\
\hline & & No & $4.67(2.79,5.00)$ & & \\
\hline & \multirow{2}{*}{ COVID-19 2} & Yes & $4.76(3.33,5.00)$ & -0.92 & 0.361 \\
\hline & & No & $4.67(2.79,5.00)$ & & \\
\hline \multirow{2}{*}{ Simulation-based $\mathrm{PPE}^{3}$ training } & \multirow{2}{*}{\multicolumn{2}{|c|}{$\begin{array}{l}\text { Yes } \\
\text { No }\end{array}$}} & $4.76(3.33,5.00)$ & -1.99 & $<0.05$ \\
\hline & & & $4.64(2.79,5.00)$ & & \\
\hline \multicolumn{3}{|c|}{ IP awareness (range 1-5) ${ }^{4}$} & $4.75 \pm 0.28$ & 0.50 & $<0.05$ \\
\hline \multicolumn{3}{|c|}{ Organizational culture (range 1-7) 4} & $5.96 \pm 0.77$ & 0.26 & $<0.05$ \\
\hline
\end{tabular}

${ }^{1}$ MERS $=$ middle-east respiratory syndrome; ${ }^{2}$ COVID-19 $=$ coronavirus disease $2019 ;{ }^{3} \mathrm{PPE}=$ personal protective equipment; ${ }^{4}$ Evaluated using Pearson's correlation coefficient; ${ }^{5}$ Calculated using non-parametric analysis of Mann-Whitney U test due to small responses (under 30 cases) in variables of experience handling possible infected passengers or quarantined due to contact with suspected infectious passengers, or PPE training.

Table 4. Multivariate analysis of factors affecting infection prevention performance using linear regression with bootstrapping method.

\begin{tabular}{|c|c|c|c|c|c|c|}
\hline \multicolumn{7}{|c|}{$(N=177)$} \\
\hline \multirow{2}{*}{ Variables $^{1}$} & \multirow{2}{*}{ B } & \multicolumn{3}{|c|}{ Bootstrap } & \multirow{2}{*}{ Clearance } & \multirow{2}{*}{ VIF $^{4}$} \\
\hline & & SE & $95 \% \mathrm{CI}^{3}$ & $p$ & & \\
\hline Constant & 0.40 & 0.54 & $-0.75-1.41$ & 0.475 & & \\
\hline Awareness & 0.77 & 0.11 & $0.54-1.03$ & $<0.05$ & 0.84 & 1.19 \\
\hline Simulation-based PPE training 2 & 0.25 & 0.07 & $0.11-0.39$ & $<0.05$ & 0.97 & 1.03 \\
\hline Organizational culture & 0.04 & 0.05 & $-0.40-0.13$ & 0.347 & 0.79 & 1.27 \\
\hline
\end{tabular}

$\operatorname{Adj} R^{2}=0.29, \mathrm{~F}=12.84(p<0.05)$, Durbin-Watson's d $=1.97$

Kolmogorov-Smirnov normality test $Z=1.95(p=<0.05)$, Breusch Pegan test $\chi^{2}=16.80(p=<0.05)$

${ }^{1}$ Adjusting age, working period, and position; ${ }^{2}$ Dummy variable $=$ Yes or No (reference); ${ }^{3}$ Confidence interval is calculated after correcting for bias; ${ }^{4} \mathrm{VIF}=$ variance inflation factor.

\section{Discussion}

This study investigated IP performance and its related factors among cabin crew members who had come into direct contact during a flight with passengers who were confirmed or suspected cases of COVID-19. It was found that cabin crew members' IP performance during the COVID-19 pandemic was high, especially for mask-wearing. Additionally, IP awareness and simulation-based PPE training experience were found to be associated with IP performance.

The average IP performance score of cabin crew was 4.56, which was higher than that of medical staff (4.31) [33]. This may have been due to the timing of data collection. 
Unlike in previous studies, data in the present study were collected during the COVID-19 pandemic. It is likely that cabin crew members could have perceived the infection risk related to possible contact with passengers with confirmed COVID-19 cases during a flight as being more serious, thereby leading to increased IP performance. In fact, we found that cabin crew members who had experienced MERS in 2015 had better IP performance than those who had not (4.87 vs. 4.67$)$.

However, the IP performance of cabin crew members in this study was also higher than that of nurses during the COVID-19 pandemic (4.28) as reported in another study [21]. This may be related to the unique Korean organizational culture among cabin crew, as Korea has a strong collectivist organizational culture [27,28]. Furthermore, cabin crew members work as a team during flights, and all procedures related to in-flight services, including attire, need to follow company guidelines. In particular, this airline emphasizes that the team leader must check the health condition of the cabin crew, such as whether they have a fever or respiratory symptoms, and whether they are wearing a mask before and during flights. In a further sub-analysis, we found that supervisors' encouragement to comply with IP guidelines was highly correlated with IP performance among cabin crew (rho $=0.21, p<0.05$ ). As cabin crew members are influenced by their supervisor's leadership when working [38], the role of team leaders is considered to be important to ensure that cabin crew comply with IP guidelines.

We found that, among the factors related to IP performance, the average score for maskwearing was 4.73 , which was consistent with previous studies conducted with emergency room medical staff during the SARS epidemic and among the general population during the COVID-19 pandemic $[39,40]$. This may be related to the awareness that wearing a mask effectively prevents the spread of COVID-19, and that its importance has been emphasized worldwide [41,42]. In fact, even before airlines provided PPE to cabin crew members, they were asked to wear PPE (e.g., goggles, aprons, gloves) during flight duty, due to concerns regarding in-flight infection. A previous study reported that wearing a mask could reduce the risk of respiratory virus transmission by $65 \%$ overall, and by $96 \%$ specifically for COVID-19 (SARS 74\%, influenza 55\%) [43].

Notably, we found that the average $\mathrm{HH}$ performance score was 4.47, which was lower than that reported for medical staff (4.67) during the COVID-19 pandemic [44]. This could potentially be related to a misconception among crew members that contaminated hands are a lesser source of respiratory infection than droplets [45]. Cabin crew, who are nonmedical personnel, may have perceived $\mathrm{HH}$ as less important for preventing respiratory infections. However, this is unlikely, as $\mathrm{HH}$ awareness was significantly higher than $\mathrm{HH}$ performance. This discrepancy may have been due to the cabin environment being inadequate for performing frequent $\mathrm{HH}$. Since the cabin crew members share a limited number of bathrooms with passengers, it could be difficult for them to regularly wash their hands with soap and water during flights. This was supported by a previous study, which found that poor sink accessibility is related to decreased hand washing [46]. To compensate for this, the airline equipped all galleys with hand sanitizer and emphasized $\mathrm{HH}$; however, it still may not have been easy to perform $\mathrm{HH}$ during flight duty. Moreover, the cabin crew might be reluctant to use alcohol-based hand sanitizer because it can dry out the skin [47], and the incidence of dermatitis may increase due to frequent use of hand sanitizer in the cabin's low-humidity environment, compared to on the ground [47,48]. Since HH is crucial to preventing infection transmission [49], it is necessary for airlines to provide hand moisturizer as well as hand sanitizer, and emphasize its use, to avoid dermatitis and improve $\mathrm{HH}$ performance. In addition, to prevent infection, this airline has introduced various procedures, such as entry screening, temperature screening, wearing masks, and social distancing, which were implemented by most airlines. Moreover, cabin crew regularly disinfect sanitary facilities during flight [30].

We found that simulation-based PPE training experience and IP awareness were related to IP performance among cabin crew. Further, although organizational culture was correlated with IP performance $(r=0.26, p<0.05)$, this was not found in the multivariate 
analysis. This could be because IP awareness $(r=0.50, p<0.05)$ was more related to IP performance than organizational culture. Therefore, IP performance could be more effectively increased through IP awareness than through organizational culture.

In this study, simulation-based PPE training experience was associated with IP performance among cabin crew. This was consistent with previous studies in which practical training was found to improve IP performance among medical staff during the COVID-19 pandemic $[23,50]$. This airline operated chartered flights to Wuhan, China, in January 2020, and to Milan, Italy, in March 2020. The cabin crew onboard these chartered flights received training directly from epidemiological intelligence service officers on how to put on and remove PPE, such as Level D protective suits, N95 masks, gloves, and goggles. It has been reported that simulation-based education, including specific training in IP performance, is more effective than lecture-based education focused on knowledge transfer [24]. In fact, we found that cabin crew members with simulation-based PPE training experience demonstrated $\mathrm{HH}$ performance after removing a Level D protective suit that was significantly superior to what is generally seen in non-medical personnel, although average $\mathrm{HH}$ performance was still low for all participants. Self-contamination of one's hands after removing PPE inappropriately has reportedly occurred among medical staff [51,52]. It is, therefore, important for cabin crew members to perform $\mathrm{HH}$ to reduce infection risk after removing PPE. Thus, regular training is necessary to prepare for any outbreaks, and PPE training for cabin crew could be helpful in developing and employing simulation-based strategies.

In this study, IP awareness showed a strong positive correlation with IP performance $(\mathrm{r}=0.50, p<0.05)$, and remained a significant factor affecting IP performance in the multivariate analysis. These results were consistent with those of previous studies conducted among medical staff $[19,49]$. Among medical staff, having IP awareness was reported to contribute to reduced infection rates in hospitals by facilitating reflection on IP practices, willingness to engage in IP improvement programs, and increasing knowledge levels [53]. IP awareness is considered to be important for cabin crew to retain their ability to introduce and improve safety behaviors to prevent unsafe situations during flight [54]. IP awareness could be improved through IP training [19]; however, cabin crew members had few opportunities to develop skills and attend structural educational courses on preparedness for endemic or epidemic infectious diseases, such as malaria, yellow fever, Ebola, and COVID19 [55]. According to the Aviation Act, cabin crew should receive initial and periodic qualification training for flight duty [56,57]. These programs need to include specific IP performance training to improve awareness among cabin crew regarding infectious diseases. Further, it is necessary to conduct regular trainings to raise IP awareness among cabin crew to improve IP performance, thus leading to reduced in-flight infection transmission.

The results suggest that airlines must realize the importance of cabin crew's IP behavior to prevent global transmission of emerging infectious diseases such as COVID-19, and to offer administrative support in the form of providing hand sanitizer and PPE, as well as cabin crew's training regarding PPE, after establishment of the airline's infection control policy.

This study provides a new perspective on IP performance among cabin crew members; however, it also has a few limitations. First, there can be limitations in generalizing the results, as all participants were from one airline. Second, there is a possibility of overestimation due to the use of self-report measures. Third, experience of handling passengers with COVID-19 or MERS could not have reached significance, as few participants reported having such experience.

\section{Conclusions}

In this study, cabin crew demonstrated a high level of IP performance. In particular, mask-wearing was high compared to $\mathrm{HH}$. Additionally, the study identifies that simulationbased PPE training experience and IP awareness are related to IP performance. To prepare for potential future pandemics which could be globally transmitted by air travel, it is necessary for airlines to develop a positive organizational culture, provide regular IP training 
for cabin crew members, and offer administrative support, such as proper equipment (e.g., PPE, hand sanitizer, and hand moisturizer). Additionally, it is the responsibility of cabin crew members to make an effort to improve IP performance.

Author Contributions: J.R.: conceptualization, data curation, formal analysis, software, methodology, validation, writing—original draft. J.K.: Conceptualization, data curation, formal analysis, investigation, methodology, validation, and writing of original draft. S.C.-K.: Writing — review \& editing, supervision, project administration. All authors have read and agreed to the published version of the manuscript.

Funding: This research received no external funding.

Institutional Review Board Statement: The study was conducted according to the guidelines of the Declaration of Helsinki, and approved by the Institutional Review Board of the Seoul National University (IRB No. 2009/001-014, 11 September 2020).

Informed Consent Statement: Informed consent was obtained from subjects involved in the study via the online survey form.

Data Availability Statement: The data are not publicly available due to privacy or ethical restrictions.

Conflicts of Interest: The authors declare no conflict of interest.

\section{References}

1. Kemenesi, G.; Zeghbib, S.; Somogyi, B.A.; Tóth, G.E.; Bányai, K.; Solymosi, N.; Szabo, P.M.; Szabó, I.; Bálint, Á.; Urbán, P.; et al. Multiple SARS-CoV-2 Introductions Shaped the Early Outbreak in Central Eastern Europe: Comparing Hungarian Data to a Worldwide Sequence Data-Matrix. Viruses. 2020, 12, 1401. [CrossRef]

2. Weekly Epidemiological Update 29 December 2020. Available online: https://www.who.int/publications/m/item/weeklyepidemiological-update---29-december-2020 (accessed on 10 January 2021).

3. Tarro, G. The New Coronavirus from the Chinese City of Wuhan. Int. J. Recent. Sci. Res. 2020, 11, 36901-36902.

4. Liu, J.Y.; Chen, T.J.; Hwang, S.J. Analysis of Imported Cases of COVID-19 in Taiwan: A Nationwide Study. Int. J. Environ. Res. Public. Health. 2020, 17, 3311. [CrossRef]

5. Killeen, G.F; Kiware, S.S. Why Lockdown? Why National Unity? Why Global Solidarity? Simplified Arithmetic Tools for Decision-Makers, Health Professionals, Journalists and the General Public to Explore Containment Options for the 2019 Novel Coronavirus. Infect. Dis. Model. 2020, 5, 442-458. [CrossRef]

6. Bae, S.H.; Shin, H.; Koo, H.Y.; Lee, S.W.; Yang, J.M.; Yon, D.K. Asymptomatic Transmission of SARS-CoV-2 on Evacuation Flight. Emerg. Infect. Dis. 2020, 26, 2705-2708. [CrossRef] [PubMed]

7. Kamata, K.; Jindai, K.; Ide, K.; Funaki, T.; Saito, H.; Takeshita, N.; Ohmagari, N.; Hinoshita, E.; Asanuma, K. The Flight Evacuation Mission for COVID-19 from Wuhan, China to Tokyo, Japan from 28 January to 17 February 2020. Jpn. J. Infect. Dis. 2020. In press. [CrossRef]

8. Browne, A.; Ahmad, S.S.; Beck, C.R.; Nguyen-Van-Tam, J.S. The Roles of Transportation and Transportation Hubs in the Propagation of Influenza and Coronaviruses: A Systematic Review. J. Travel. Med. 2016, 23, tav002. [CrossRef]

9. Grout, A.; Howard, N.; Coker, R.; Speakman, E.M. Guideline, Law, and Governance: Disconnects in the Control of Airlineassociated Infectious Disease. Lancet Infect. Dis. 2017, 17, e118-e122. [CrossRef]

10. Choi, E.M.; Chu, D.K.W.; Cheng, P.K.C.; Tsang, D.N.C.; Peiris, M.; Bausch, D.G.; Poon, L.L.M.; Watson-Jones, D. In-Flight Transmission of SARS-CoV-2. Emerg. Infect. Dis. 2020, 26, 2713-2716. [CrossRef] [PubMed]

11. Yang, N.; Shen, Y.; Shi, C.; Ma, A.H.Y.; Zhang, X.; Jian, X.; Wang, L.; Shi, J.; Wu, C.; Li, G.; et al. In-Flight Transmission Cluster of COVID-19: A Retrospective Case Series. Infect. Dis. 2020, 52, 891-901. [CrossRef] [PubMed]

12. Han, Z.; To, G.N.; Fu, S.C.; Chao, C.Y.; Weng, W.; Huang, Q. Effect of Human Movement on Airborne Disease Transmission in an Airplane Cabin: Study Using Numerical Modeling and Quantitative Risk Analysis. BMC Infect. Dis. 2014, 14, 434. [CrossRef]

13. Hertzberg, V.S.; Weiss, H.; Elon, L.; Si, W.; Norris, S.L.; FlyHealthy Research Team. Behaviors, Movements, and Transmission of Droplet-Mediated Respiratory Diseases During Transcontinental Airline Flights. Proc. Natl. Acad. Sci. USA 2018, 115, 3623-3627. [CrossRef] [PubMed]

14. Peddle, M.B.; Smith, J.A. Hazard Control for Communicable Disease Transport at Ornge. Can. J. Emerg. Med. 2020, 22, S79-S83. [CrossRef]

15. World Health Organization. Management of Ill Travellers at Points of Entry-International Airports, Seaports and Ground Crossings-In the Context of COVID-19 Outbreak: Interim Guidance; World Health Organization: Geneva, Switzerland, 16 February 2020.

16. Gupta, J.K.; Lin, C.H.; Chen, Q. Risk Assessment of Airborne Infectious Diseases in Aircraft Cabins. Indoor. Air. 2012, 22, 388-395. [CrossRef] [PubMed]

17. Freedman, D.O.; Wilder-Smith, A. In-Flight Transmission of SARS-CoV-2: A Review of the Attack Rates and Available Data on the Efficacy of Face Masks. J. Travel. Med. 2020, 27, taaa178. [CrossRef] 
18. Oh, J.E.; Park, J.Y. Influencing Factors on Performance for Standard Precaution of Healthcare Workers of General Hospital for Infection Control. J. Digit. Converg. 2018, 16, 231-249.

19. Park, Y.M.; Park, H.-S.; Park, K.-Y. Factors Influencing Level of Awareness and Compliance with Vancomycin-resistant Enterococcus Infection Control Among Nurses in Intensive Care Units. J. Korean Acad. Fundam. Nurs. 2008, 15, 531-538.

20. Moore, D.; Gamage, B.; Bryce, E.; Copes, R.; Yassi, A.; BC Interdisciplinary Respiratory Protection Study Group. Protecting Health Care Workers from SARS and Other Respiratory Pathogens: Organizational and Individual Factors that Affect Adherence to Infection Control Guidelines. Am. J. Infect. Control 2005, 33, 88-96. [CrossRef]

21. Al-Dossary, R.; Alamri, M.; Albaqawi, H.; Al Hosis, K.; Aljeldah, M.; Aljohan, M.; Aljohani, K.; Almadani, N.; Alrasheadi, B.; Falatah, R.; et al. Awareness, Attitudes, Prevention, and Perceptions of COVID-19 Outbreak Among Nurses in Saudi Arabia. Int. J. Environ. Res. Public. Health. 2020, 17, 8269. [CrossRef]

22. Kim, Y. Nurses' Experiences of Care for Patients with Middle East Respiratory Syndrome-Coronavirus in South Korea. Am. J. Infect. Control. 2018, 46, 781-787. [CrossRef]

23. Tan, W.; Ye, Y.; Yang, Y.; Chen, Z.; Yang, X.; Zhu, C.; Chen, D.; Tan, J.; Zhen, C. Whole-Process Emergency Training of Personal Protective Equipment Helps Healthcare Workers Against COVID-19: Design and Effect. J. Occup. Environ. Med. 2020, 62, 420-423. [CrossRef]

24. Cho, S.S.; Kim, K.M.; Lee, B.Y.; Park, S.A. The Effects of Simulation-based Infection Control Training on the Intensive Care Unit Nurses' Perception, Clinical Performance, and Self-Efficacy of Infection Control. J. Korean. Clin. Nurs. Res. 2012, 18, 381-390.

25. Suess, T.; Remschmidt, C.; Schink, S.B.; Schweiger, B.; Nitsche, A.; Schroeder, K.; Doellinger, J.; Milde, J.; Haas, W.; Koehler, I.; et al. The Role of Facemasks and Hand Hygiene in the Prevention of Influenza Transmission in Households: Results from a Cluster Randomised Trial; Berlin, Germany, 2009-2011. BMC. Infect. Dis. 2012, 12, 26. [CrossRef]

26. Chung, K.I. A Comparative Study of the Organizational Culture of the Hotels Between Korea and United States. Korean. Hosp. Tourism. Acad. 2001, 10, 185-200.

27. Taylor, I.; Brotheridge, C.M. Cultural Differences in Emotional Labor in Flight Attendants. In Individual and Organizational Perspectives on Emotion Management and Display; Härtel, C.E.J., Zerbe, W.J., Ashkanasy, N.M., Eds.; Emerald Group Publishing Limited: Bingley, UK, 2006; pp. 167-191.

28. Ha, A.-N.; Lee, H.-S. The Effects of Organizational Culture Within Airline's Crew Team on Organizational Commitment the Team and Customer Orientation. Tour. Res. 2017, 42, 193-220.

29. van Buijtene, A.; Foster, D. Does a Hospital Culture Influence Adherence to Infection Prevention and Control and Rates of Healthcare Associated Infection? A Literature Review. J. Infect. Prev. 2019, 20, 5-17. [CrossRef]

30. Bielecki, M.; Patel, D.; Hinkelbein, J.; Komorowski, M.; Kester, J.; Ebrahim, S. Air Travel and COVID-19 Prevention in the Pandemic and Peri-pandemic Period: A Narrative Review. Travel. Med. Infect. Dis. 2021, 39, 101915. [CrossRef]

31. Hong, S.; Kwon, Y.-S.; Park, H. Nursing Students' Awareness and Performance on Standard Precautions of Infection Control in the Hospital. J. Korean. Acad. Soc. Nurs. Edu. 2012, 18, 293-302. [CrossRef]

32. Polit, D.F.; Beck, C.T. The Content Validity Index: Are You Sure You Know What's Being Reported? Critique and Recommendations. Res. Nurs. Health 2006, 29, 489-497. [CrossRef]

33. Kim, H.; Park, H.-R. The Effects of Organizational Culture for Infection Control and Self-Efficacy on Compliance with Standard Precautions of Emergency Room Nurses. J. Korean. Biol. Nurs. Sci. 2019, 21, 46-53. [CrossRef]

34. Zhu, J.; Jing, P. The Analysis of Bootstrap Method in Linear Regression Effect. J. Math. Res. 2010, 2, 64. [CrossRef]

35. Cohen, J. Statistical Power Analysis. Curr. Dir. Psychol. Sci. 1992, 1, 98-101. [CrossRef]

36. Nahm, F.S. Understanding Effect Sizes. Hanyang. Med. Rev. 2015, 35, 40-43. [CrossRef]

37. Huang, C.-C.L.; Jou, Y.-J.; Cho, H.-J. VIF-Based Adaptive Matrix Perturbation Method for Heteroskedasticity-Robust Covariance Estimators in the Presence of Multicollinearity. Commun. Stat. Theor. Methods. 2017, 46, 3255-3263. [CrossRef]

38. Jung, H.S.J.; Yoon, H.H. Why Is Supervisor Leadership Important? The Effects of Leadership Styles on Work Engagement and Innovative Behavior Among Flight Attendant. Int. J. Tourism. Hosp. Res. 2020, 34, 75-90. [CrossRef]

39. Bazaid, A.S.; Aldarhami, A.; Binsaleh, N.K.; Sherwani, S.; Althomali, O.W. Knowledge and Practice of Personal Protective Measures During the COVID-19 Pandemic: A Cross-Sectional Study in Saudi Arabia. PLoS ONE 2020, 15, e0243695. [CrossRef]

40. Parker, M.J.; Goldman, R.D. Paediatric Emergency Department Staff Perceptions of Infection Control Measures Against Severe Acute Respiratory Syndrome. Emerg. Med. J. 2006, 23, 349-353. [CrossRef]

41. Jeong, G.; Lee, T.R.; Hwang, S.Y.; Cha, W.C.; Shin, T.G.; Sim, M.S.; Jo, I.J.; Song, K.J.; Rhee, J.E.; Jeong, Y.K. Emergency of Department Workers' Perceptions of Effectiveness and Reported Compliance of Infection Control Measures After Middle East Respiratory Syndrome Outbreaks. J. Korean. Soc. Emerg. Med. 2016, 27, 328-335.

42. Tso, R.V.; Cowling, B.J. Importance of Face Masks for COVID-19: A Call for Effective Public Education. Clin. Infect. Dis. 2020, 71, 2195-2198. [CrossRef]

43. Liang, M.; Gao, L.; Cheng, C.; Zhou, Q.; Uy, J.P.; Heiner, K.; Sun, C. Efficacy of Face Mask in Preventing Respiratory Virus Transmission: A Systematic Review and Meta-Analysis. Travel. Med. Infect. Dis. 2020, 36, 101751. [CrossRef]

44. Assefa, D.; Melaku, T.; Bayisa, B.; Alemu, S. Knowledge, Attitude and Self-Reported Performance and Challenges of Hand Hygiene Using Alcohol-Based Hand Sanitizers Among Healthcare Workers During COVID-19 Pandemic at a Tertiary Hospital: A Cross-Sectional Study. Infect. Drug. Resist. 2021, 14, 303-313. [CrossRef] 
45. Gautret, P.; Soula, G.; Parola, P.; Brouqui, P. Hajj Pilgrims' Knowledge About Acute Respiratory Infections. Emerg. Infect. Dis. 2009, 15, 1861-1862. [CrossRef]

46. Deyneko, A.; Cordeiro, F.; Berlin, L.; Ben-David, D.; Perna, S.; Longtin, Y. Impact of Sink Location on Hand Hygiene Compliance After Care of Patients with Clostridium difficile Infection: A Cross-Sectional Study. BMC. Infect. Dis. 2016, 16, 203. [CrossRef]

47. Beiu, C.; Mihai, M.; Popa, L.; Cima, L.; Popescu, M.N. Frequent Hand Washing for COVID-19 Prevention Can Cause Hand Dermatitis: Management Tips. Cureus 2020, 12, e7506. [CrossRef]

48. Leggat, P.A.; Smith, D.R. Dermatitis and Aircrew. Contact. Dermatitis. 2006, 54, 1-4. [CrossRef]

49. Pittet, D.; Simon, A.; Hugonnet, S.; Pessoa-Silva, C.L.; Sauvan, V.; Perneger, T.V. Hand Hygiene Among Physicians: Performance, Beliefs, and Perceptions. Ann. Intern. Med. 2004, 141, 1-8. [CrossRef]

50. Díaz-Guio, D.A.; Ricardo-Zapata, A.; Ospina-Velez, J.; Gómez-Candamil, G.; Mora-Martinez, S.; Rodriguez-Morales, A.J. Cognitive Load and Performance of Health Care Professionals in Donning and Doffing PPE Before and After a Simulation-Based Educational Intervention and Its Implications During the COVID-19 Pandemic for Biosafety. Infez. Med. 2020, 28, 111-117.

51. Okamoto, K.; Rhee, Y.; Schoeny, M.; Lolans, K.; Cheng, J.; Reddy, S.; Weinstein, R.A.; Hayden, M.K.; Popovich, K.J.; Centers for Disease Control and Prevention Epicenters Program. Impact of Doffing Errors on Healthcare Worker Self-Contamination When Caring for Patients on Contact Precautions. Infect. Control. Hosp. Epidemiol. 2019, 40, 559-565. [CrossRef]

52. Poller, B.; Hall, S.; Bailey, C.; Gregory, S.; Clark, R.; Roberts, P.; Tunbridge, A.; Poran, V.; Crook, B.; Evans, C. “VIOLET”: A Fluorescence-Based Simulation Exercise for Training Healthcare Workers in the Use of Personal Protective Equipment. J. Hosp. Infect. 2018, 99, 229-235. [CrossRef]

53. Agarwal, N.; Agarwal, P.; Querry, A.; Mazurkiewicz, A.; Tempel, Z.J.; Friedlander, R.M.; Gerszten, P.C.; Hamilton, D.K.; Okonkwo, D.O.; Kanter, A.S. Implementation of an Infection Prevention Bundle and Increased Physician Awareness Improves Surgical Outcomes and Reduces Costs Associated with Spine Surgery. J. Neurosurg. Spine. 2018, 29, 108-114. [CrossRef]

54. Rhoden, S.; Ralston, R.; Ineson, E.M. Cabin Crew Training to Control Disruptive Airline Passenger Behavior: A Cause for Tourism Concern? Tour. Manag. 2008, 29, 538-547. [CrossRef]

55. Albayat, S.; Bansal, D.; Kokku, S.B.; Al-Romaihi, H.; Khogali, H.; Farag, E. Assessment of Cabin Crew Awareness About Malaria in a Major Airline. Mediterr. J. Hematol. Infect. Dis. 2019, 11, e2019049. [CrossRef] [PubMed]

56. Kim, J.H.; Choi-Kwon, S.; Park, Y.H. Comparison of Inflight First Aid Performed by Cabin Crew Members and Medical Volunteers. J. Travel. Med. 2017, 24, taw091. [CrossRef] [PubMed]

57. Paethrangsi, N. Competency Training of Initial Crew Resource Management Course for Cabin Crew. IOP Conf. Ser. Mater. Sci. Eng. 2020, 918, 012179. [CrossRef] 